\title{
Viral DNA Prefers to Be Compartmentalized
}

$\mathrm{V}$

Tiruses come in a range of shapes and sizes. from the tiny, icosahedral rhinovirus (common cold) to the much larger, brick-shaped orthopoxvirus (smallpox). How DNA is packed inside a virus, however, remains a controversial question. In a recent work published in Physical Review Letlers, researchers from the Institute of Physics (IoP), Chinese Academy of Sciences and the University of the Chinese Academy of Sciences (UCAS) addressed this packing problem with a combination of analytical theory and simulations.

They postulated a simplified model of an elastic filament confined to a sphere with three competing interactions: elasticity, which prefers the filament to be straight; excluded volume, which prevents the filament from overlapping with itself; and the spherical confinement, which frustrates the other two interaction terms. This simple model gives rise to a surprisingly great deal of complexity. They observe that at high densities the preferred structure is a complex arrangement of intertwined rings and that compartmentalization into multiple domains is always preferred to a single. donutshaped domain proposed in previous works.

Multidomain ordering is known from the structure of chromatin, where the complex protein-DNA interactions drive the segregation of DNA. Here, the researchers have shown that it can emerge in a much simpler and smaller system, in the absence of specific structural constraints. In making the leap to predict the exact packing in real viruses, the specific interactions between the DNI and the viral shell, and within the DNA molecule itself. should naturally be considered. However, their results give a general context in which viral DNA packing can be discussed, offering fresh insight into the fundamental mechanisms that govern this process.

\section{Contact}

Institute of Physics, CAS

Dr. Jure Dobnikar

Jd489@cam.ac.uk
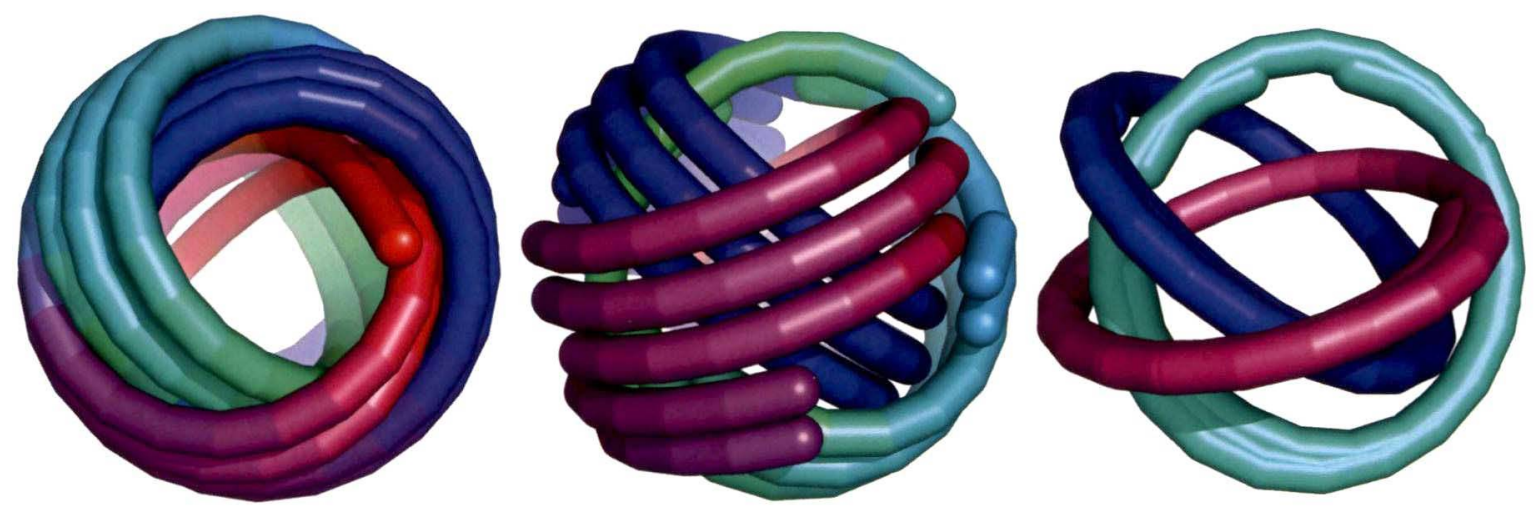

Compartmentalization of confined viral DNA. (Images by J.D. Farrell, loP)

\section{Reference}

Tine Curk, James D. Farrell, Jure Dobnikar, Rudi Podgornik, Spontaneous Domain Formation in Spherically-Confined Elastic Filaments, Phys. Rev. Lett. 123047801 (2019) 